\title{
Compensation for unmatched uncertainty with adaptive RBF network
}

\author{
S.W. Wang ${ }^{1 *}$, D.W. Yu ${ }^{2}$, D.L. Yu ${ }^{3}$ \\ ${ }^{1 *}$ Yuan Hang Scientific Corp. Weihai City, CHINA \\ ${ }^{2}$ Department of Engineering, Northeast University at Qinhuangdao, CHINA \\ ${ }^{3}$ Control Systems Research Group, School of Engineering, Liverpool John Moores University, Liverpool, UK \\ "Corresponding Author: e-mail: d.yu@ljmu.ac.uk, Tel +44-151-2312360,
}

\begin{abstract}
Robust control for nonlinear uncertain systems has been solved for matched uncertainty but has not been completely solved yet for unmatched uncertainty. This paper developed a new method in which an adaptive radial basis function neural network is used to compensate for the effects of unmatched uncertainty in the framework of integral sliding mode control. The stability of the whole system is guaranteed by the Lyapunov method. The adaptation algorithm of the network is also derived by the Lyapunov function so that its convergence is also guaranteed. A numerical example is used to show the effectiveness of the proposed method. Improvement against existing methods is also demonstrated.
\end{abstract}

Keywords: Integral sliding mode, neural networks, matched and unmatched uncertainty.

\section{Introduction}

Integral sliding mode control (ISMC) has attracted wide interests in nonlinear control community (Utkin \& Shi, 1996; Poznyak, Fridman \& Bejarano, 2004). It introduces an integral term into the sliding surface, which makes the system initial states start from the sliding mode and eliminates the reaching phase. Thus, ISMC enhances the robustness against matched uncertainty of the conventional SMC (Utkin, 1977; Matthews \& DeCarlo, 1988; Young, Utkin \& Ozguner, 1999; Fridman, Poznyak \& Bejarano, 2005; Niu, Ho \& Lam, 2005.). However, it is still sensitive to unmatched uncertainties that exist in many practical systems. An ISMC controlled system was developed that completely nullifies matched uncertainties, but with the unmatched uncertainty the system stability depends on the controlled nominal system and the features of the equivalent unmatched uncertainties (Cao \& Xu, 2004). Castanos \& Fridman (2006) discussed mainly how to select the optimal design matrix to ensure that the unmatched uncertainty is not amplified by the discontinuous control, but it is only suitable for a certain type of nonlinear systems with constant input matrices. The research on ISMC has been focused on how to reduce the influence of unmatched uncertainty, which is the motivation of this paper. According to the literature (Funahashi, 1989; Sanner and Slotine, 1992; Wang and Hill, 2006.), radial basis function (RBF) neural networks have showed strong universal approximation ability for unknown system nonlinearities. Therefore, a RBF network is capable of approximating and counteracting wholly or partially the term of the unmatched uncertainty in the ISMC system dynamics. Based on this basic idea, the main contribution of this paper is proposing a new integral sliding surface that includes an additional design matrix with an adaptive RBF neural network. In addition to the advantages of no reaching phase and nullifying matched uncertainties, more importantly, it compensates partially the effects of unmatched uncertainties in the system closed-loop dynamics and thus enhances the robustness compared with previous research (Cao and Xu, 2004; Castanos and Fridman, 2006). The adaptation law of the RBF network is derived using a defined Lyapunov function. Also based on Lyapunov theory, the switching gain condition is obtained to ensure the system states remaining on the designed sliding surface. In addition, many other methods for sliding mode control for systems with uncertainties have been investigated, such as Seok et al. (2009), Yinxing et al. (2008) and Wang and Yu (2008). 
This paper presents the developed method only with a numerical example. The real data experiments using the method are not included as the research is not come to that stage and further investigation will be reported when results are available. Also, the unknown disturbance is not dealt with in this study. This may be tackled by neural network modeling in on-line mode but the disturbance structure should be known for easy implementation.

\section{Problem Statement}

Consider the following nonlinear uncertain system

$$
\dot{x}=f(x)+B(x)\left\{\left[I+\Delta B_{m}(x)\right] u+\Delta f_{m}(x)\right\}+\Delta f_{u}(x)
$$

where $x(t) \in \Omega \subset R^{n}$ is the measurable state vector and $\Omega$ is an arbitrary large compact set, $u(t) \in R^{m}$ is the control vector, $f(x) \in R^{n}$ and $B(x) \in R^{n \times m}$ are known nonlinear functions and $\operatorname{rank}\{B(x)\}=m . \Delta f_{m}(x)$ and $\Delta B_{m}(x)$ are the matched uncertainties. The unknown continuous function $\Delta f_{u}(x)$ is the unmatched uncertainty. As $\Delta f_{u}(x)$ represents mainly the deviation of $f(x)$ while $\Delta f_{m}(x)$ represents the deviation of $\Delta B_{m}(x)$, they are functions of $x(t)$ instead of time $t$. It is assumed that all system uncertainties are bounded, i.e. there exist $\left\|\Delta f_{m}(x)\right\| \leq \rho_{m}(x),\left\|\Delta B_{m}(x)\right\| \leq 1-\varepsilon_{b}$ and $\left\|\Delta f_{u}(x)\right\| \leq \rho_{u}(x)$ where $\rho_{m}(x)$ and $\rho_{u}(x)$ are known nonnegative nonlinear functions and $\varepsilon_{b}$ is a positive constant and $\varepsilon_{b}<1$.

Assumption 1. The known nominal nonlinear plant of the system (1) is

$$
\dot{x}=f(x)+B(x) u_{n}(x)
$$

which is globally asymptotically stabilizable via a nominal control $u_{n}(x)$, i.e., there is a Lyapunov function $V(x)$, such that its first-order partial derivative satisfies

$$
\begin{gathered}
\gamma_{1}(\|x\|) \leq V(x) \leq \gamma_{2}(\|x\|) \\
\dot{V}(x)=\left(\frac{\partial V}{\partial x}\right)^{T}\left[f(x)+B(x) u_{n}(x)\right] \leq-\gamma(\|x\|)
\end{gathered}
$$

Here, $\gamma_{1}, \gamma_{2}: R^{+} \rightarrow R^{+}$are class $K_{\infty}$ functions, $\gamma: R^{+} \rightarrow R^{+}$is defined as $\gamma(\|x\|)=\beta\|x\|$, where $\beta>0$.

\section{New Integral Sliding Surface}

In this paper, a new integral-type sliding surface is proposed as

$$
S(x)=D x-D x_{0}-\int_{t_{0}}^{t}\left[D f(x)+D B(x) u_{n}(x)-D B(x) \hat{f}_{N N}(x)\right] d \tau=0
$$

where $x_{0}$ is the state vector at time $t_{0}, D \in R^{m \times n}$ satisfies that $D B(x)$ is uniformly invertible. $\hat{f}_{N N}(x) \in \mathfrak{R}^{m}$ is a RBF network in the following form (a RBF network is used here rather than a MLP or other types because the simplicity of RBF in the structure and in training is considered):

$$
\hat{f}_{N N}(x)=\sum_{i=1}^{n_{h}} \hat{w}_{i} \exp \left[-\frac{\left\|x-c_{i}\right\|^{2}}{\sigma_{i}^{2}}\right]
$$

where $n_{h}$ is the number of network centers, $\hat{w}_{i}(t) \in \mathfrak{R}^{m}$ is the network weight, $c_{i}=\left[c_{1 i}, c_{2 i}, \cdots, c_{n i}\right]^{T} \in R^{n}$ are the network center vector and $\sigma_{i}$ is the network width. The last term of the integral part in the sliding surface (5) can be treated as a design vector $g(x) \in R^{m}$, i.e.

$$
g(x)=-D B(x) \hat{f}_{N N}(x)=-D B(x) \sum_{i=1}^{n_{h}} \hat{w}_{i} \exp \left[-\frac{\left\|x-c_{i}\right\|^{2}}{\sigma_{i}^{2}}\right]
$$

Take the first derivative of the sliding surface $S(x)$ in (5) 


$$
\dot{S}(x)=D B(x)\left\{\left[I+\Delta B_{m}(x)\right] u+\Delta f_{m}(x)-u_{n}(x)\right\}+D \Delta f_{u}(x)-g(x)
$$

In the sliding mode, $S(x)=\dot{S}(x)=0$ and $x(t)=x_{d}(t)$. Here subscript $d$ denotes the state vector in the sliding mode. The equivalent control law is

$$
u_{e q}\left(x_{d}\right)=\left[I+\Delta B_{m}\left(x_{d}\right)\right]^{-1}\left\{-\left[D B\left(x_{d}\right)\right]^{-1}\left[D \Delta f_{u}(x)-g(x)\right]-\Delta f_{m}\left(x_{d}\right)+u_{n}\left(x_{d}\right)\right\}
$$

Substituting the above equation into (1), one obtains the closed-loop dynamics of the sliding surface

$$
\dot{x}_{d}=f\left(x_{d}\right)+B\left(x_{d}\right) u_{n}\left(x_{d}\right)+\left\{I-B\left(x_{d}\right)\left[D B\left(x_{d}\right)\right]^{-1} D\right\} \Delta f_{u}\left(x_{d}\right)+B\left(x_{d}\right)\left[D B\left(x_{d}\right)\right]^{-1} g\left(x_{d}\right)
$$

Then, define a vector $\phi_{u}(x) \in R^{n}$ as follows,

$$
\phi_{u}(x)=\left[\begin{array}{l}
\phi_{u 1}(x) \\
\phi_{u 2}(x)
\end{array}\right]=Q^{-1}(x)\left\{I-B(x)[D B(x)]^{-1} D\right\} \Delta f_{u}(x)
$$

with $\phi_{u 1}(x) \in \mathfrak{R}^{m}$ and $\phi_{u 2}(x) \in \mathfrak{R}^{n-m}$, where $Q(x) \in \mathfrak{R}^{n \times n}$ is an orthogonal matrix from the QR decomposition of $B(x)$,

$$
Q(x)\left[\begin{array}{c}
R(x) \\
0
\end{array}\right]=B(x)
$$

By dividing $Q(x)$ into two sub-matrices $Q_{1}(x) \in \mathfrak{R}^{n \times m}$ and $Q_{2}(x) \in \mathfrak{R}^{n \times(n-m)}$, it is obtained that

$$
Q(x)\left[\begin{array}{l}
\phi_{u 1}(x) \\
\phi_{u 2}(x)
\end{array}\right]=\left[\begin{array}{ll}
Q_{1}(x) & Q_{2}(x)
\end{array}\right]\left[\begin{array}{l}
\phi_{u 1}(x) \\
\phi_{u 2}(x)
\end{array}\right]=\left\{I-B(x)[D B(x)]^{-1} D\right\} \Delta f_{u}(x)
$$

Considering (7) and (13), the closed-loop dynamics become

$$
\dot{x}_{d}=f\left(x_{d}\right)+B\left(x_{d}\right) u_{n}\left(x_{d}\right)+Q_{1}\left(x_{d}\right) \phi_{u 1}\left(x_{d}\right)+Q_{2}\left(x_{d}\right) \phi_{u 2}\left(x_{d}\right)-B\left(x_{d}\right) \hat{f}_{N N}(x)
$$

Then, the problem becomes: how to design the RBF network $\hat{f}_{N N}(x)$ to reduce the influence of the unmatched uncertainty $\Delta f_{u}(x)$ on the closed-loop dynamics.

Theorem 1. The closed-loop dynamics of the nonlinear system (1) on the integral sliding surface (5)

$$
\dot{x}_{d}=f\left(x_{d}\right)+B\left(x_{d}\right) u_{n}\left(x_{d}\right)+Q_{2}\left(x_{d}\right) \phi_{u 2}\left(x_{d}\right)
$$

is globally asymptotically stable for

$$
\beta>\left\|\phi_{u 2}\left(x_{d}\right)\right\|+\left\|B\left(x_{d}\right)\right\| \varepsilon \quad \forall x_{d} \in R^{n}, \text { when } \quad x_{d} \neq 0
$$

if the RBF network weights are adapted as follows

$$
\hat{w}_{i}(t)=\hat{w}_{i}\left(t_{0}\right)+\int_{t_{0}}^{t} \eta \cdot x_{d}^{T} B\left(x_{d}\right) \exp \left[-\frac{\left\|x-c_{i}\right\|^{2}}{\sigma_{i}^{2}}\right] d \tau, \quad i=1, \cdots, n_{h}
$$

Where the adaptation parameter $\eta$ in (17) is a positive constant chosen by users and $\varepsilon$ in (16) is a positive constant.

Proof. Considering the unmatched uncertainty $\Delta f_{u}(x)$ is a bounded continuous function, thus the unknown function $R^{-1}(x) \phi_{u 1}(x): \Omega \rightarrow R^{m}$ containing one part of unmatched uncertainty is continuous over a compact set $\Omega \subset \Re^{n}$. According to the universal approximation property of RBF networks (Funahashi 1989; Sanner and Slotine 1992; Wang and Hill 2006), the function can be approximated by a RBF network $f_{N N}(x)$ to arbitrary any accuracy using sufficient center number $n_{h}$, i.e.

$$
R^{-1}(x) \phi_{u 1}(x)=f_{N N}(x)+e(x)=\sum_{i=1}^{n_{h}} w_{i} \exp \left[-\frac{\left\|x-c_{i}\right\|^{2}}{\sigma_{i}^{2}}\right]+e(x) \quad \forall x \in \Omega
$$

where $R(x) \in \mathfrak{R}^{m \times m}$ is an upper triangular matrix from the QR decomposition of $B(x), w_{i}$ is the ideal constant weight, the approximation error $e(x)$ satisfies $|e(x)|<\varepsilon$.

The RBF network $\hat{f}_{N N}(x)$ is used as an estimator of $f_{N N}(x)$ by adapting its weight $\hat{w}_{i}(t)$ to converge to the ideal constant value $w_{i}$ with the weight estimation error $\tilde{w}_{i}(t)$, i.e. $w_{i}=\hat{w}_{i}(t)+\widetilde{w}_{i}(t)$. Thus, equation (18) becomes 


$$
R^{-1}(x) \phi_{u 1}(x)=\sum_{i=1}^{n_{h}}\left[\hat{w}_{i}+\tilde{w}_{i}\right] \exp \left[-\frac{\left\|x-c_{i}\right\|^{2}}{\sigma_{i}^{2}}\right]+e(x)
$$

Define a Lyapunov function as follows

$$
V_{1}\left(x_{d}\right)=\frac{1}{2} x_{d}^{T} x_{d}+\frac{1}{2 \eta} \sum_{i=1}^{n_{h}} \widetilde{w}_{i}^{2}
$$

Its first partial derivative is

$$
\begin{aligned}
\dot{V}_{1}\left(x_{d}\right) & =x_{d}^{T} \dot{x}_{d}-\frac{1}{\eta} \sum_{i=1}^{n_{h}} \tilde{w}_{i} \dot{\hat{w}}_{i} \\
& =x_{d}^{T}\left\{f\left(x_{d}\right)+B\left(x_{d}\right) u_{n}\left(x_{d}\right)+Q\left(x_{d}\right) \phi_{u}\left(x_{d}\right)-B\left(x_{d}\right) \hat{f}_{N N}(x)\right\}-\frac{1}{\eta} \sum_{i=1}^{n_{h}} \tilde{w}_{i} \dot{\hat{w}}_{i}
\end{aligned}
$$

According to (13), (19) and (12), the term $Q\left(x_{d}\right) \phi_{u}\left(x_{d}\right)$ is represented as follows,

$$
Q\left(x_{d}\right) \phi_{u}\left(x_{d}\right)=Q_{1}\left(x_{d}\right) \phi_{u 1}\left(x_{d}\right)+Q_{2}\left(x_{d}\right) \phi_{u 2}\left(x_{d}\right)
$$

$$
\begin{aligned}
& =\left[\begin{array}{ll}
Q_{1}\left(x_{d}\right) & Q_{2}\left(x_{d}\right)
\end{array}\right] \cdot\left[\begin{array}{c}
R\left(x_{d}\right) \\
0
\end{array}\right] R^{-1}\left(x_{d}\right) \phi_{u 1}\left(x_{d}\right)+Q_{2}\left(x_{d}\right) \phi_{u 2}\left(x_{d}\right) \\
& =\left[\begin{array}{ll}
Q_{1}\left(x_{d}\right) & Q_{2}\left(x_{d}\right)
\end{array}\right] \cdot\left[\begin{array}{c}
R\left(x_{d}\right) \\
0
\end{array}\right]\left\{\begin{array}{l}
\left.\sum_{i=1}^{n_{h}}\left[\hat{w}_{i}+\tilde{w}_{i}\right] \exp \left[-\frac{\left\|x_{d}-c_{i}\right\|^{2}}{\sigma_{i}^{2}}\right]\right\}+Q_{2}\left(x_{d}\right) \phi_{u 2}\left(x_{d}\right) \\
+e\left(x_{d}\right)
\end{array}\right. \\
& =B\left(x_{d}\right)\left\{\hat{f}_{N N}\left(x_{d}\right)+\sum_{i=1}^{n_{h}} \tilde{w}_{i} \exp \left[-\frac{\left\|x_{d}-c_{i}\right\|^{2}}{\sigma_{i}^{2}}\right]+e\left(x_{d}\right)\right\}+Q_{2}\left(x_{d}\right) \phi_{u 2}\left(x_{d}\right)
\end{aligned}
$$

Then, it follows that

$$
\begin{aligned}
\dot{V}_{1}\left(x_{d}\right)=x_{d}^{T}\left[f\left(x_{d}\right)+B\left(x_{d}\right) u_{n}\left(x_{d}\right)\right]+x_{d}^{T} Q_{2}\left(x_{d}\right) \phi_{u 2}\left(x_{d}\right) \\
+x_{d}^{T} B\left(x_{d}\right)\left\{\sum_{i=1}^{n_{h}} \tilde{w}_{i} \exp \left[-\frac{\left\|x_{d}-c_{i}\right\|^{2}}{\sigma_{i}^{2}}\right]+e\left(x_{d}\right)\right\}-\frac{1}{\eta} \sum_{i=1}^{n_{h}} \tilde{w}_{i} \dot{\hat{w}}_{i} \\
=x_{d}^{T}\left[f\left(x_{d}\right)+B\left(x_{d}\right) u_{n}\left(x_{d}\right)\right]+x_{d}^{T} Q_{2}\left(x_{d}\right) \phi_{u 2}\left(x_{d}\right) \\
+x_{d}^{T} B\left(x_{d}\right) e\left(x_{d}\right)+\sum_{i=1}^{n_{h}} \tilde{w}_{i}\left\{x_{d}^{T} B\left(x_{d}\right) \exp \left[-\frac{\left\|x_{d}-c_{i}\right\|^{2}}{\sigma_{i}^{2}}\right]-\frac{1}{\eta} \dot{\hat{w}}_{i}\right\}
\end{aligned}
$$

Choosing

$$
\dot{\hat{w}}_{i}=\eta \cdot x_{d}^{T} B\left(x_{d}\right) \exp \left[-\frac{\left\|x_{d}-c_{i}\right\|^{2}}{\sigma_{i}^{2}}\right]
$$

the first partial derivative of the defined Lyapunov function becomes

$$
\dot{V}_{1}\left(x_{d}\right)=x_{d}^{T}\left[f\left(x_{d}\right)+B\left(x_{d}\right) u_{n}\left(x_{d}\right)\right]+x_{d}^{T} Q_{2}\left(x_{d}\right) \phi_{u 2}\left(x_{d}\right)+x_{d}^{T} B\left(x_{d}\right) e\left(x_{d}\right)
$$

Considering Assumption 1 and the norm of a matrix with orthonormal columns being 1, it is obtained from (25) that 


$$
\begin{aligned}
\dot{V}_{1}\left(x_{d}\right) & <-\gamma\left(\left\|x_{d}\right\|\right)+\left\|x_{d}^{T}\right\|\left\|\phi_{u 2}\left(x_{d}\right)\right\|+\left\|x_{d}^{T}\right\|\left\|B\left(x_{d}\right)\right\| \varepsilon \\
& =-\beta \cdot\left\|x_{d}\right\|+\left\|x_{d}^{T}\right\|\left\|\phi_{u 2}\left(x_{d}\right)\right\|+\left\|x_{d}^{T}\right\|\left\|B\left(x_{d}\right)\right\| \varepsilon \\
& =\left\|x_{d}^{T}\right\| \cdot\left[-\beta+\left\|\phi_{u 2}\left(x_{d}\right)\right\|+\left\|B\left(x_{d}\right)\right\| \varepsilon\right]
\end{aligned}
$$

Noticing $\left\|\phi_{u 2}\left(x_{d}\right)\right\| \leq\left\|\phi_{u}\left(x_{d}\right)\right\|=\left\|I-B\left(x_{d}\right)\left[D B\left(x_{d}\right)\right]^{-1} D\right\| \cdot\left\|\Delta f_{u}\left(x_{d}\right)\right\| \leq\left\|I-B\left(x_{d}\right)\left[D B\left(x_{d}\right)\right]^{-1} D\right\| \rho_{u}\left(x_{d}\right)$ and considering that $\left\|B\left(x_{d}\right)\right\|$ is bounded and $D$ is constant matrix, $\left\|I-B\left(x_{d}\right)\left[D B\left(x_{d}\right)\right]^{-1} D\right\| \leq \rho_{x}\left(x_{d}\right)$ is also bounded. Besides, $\varepsilon$ is a positive constant so that $\varepsilon^{\prime}=\left\|B\left(x_{d}\right)\right\| \varepsilon$ is also a positive constant. Therefore, a $\beta$ satisfying $\beta>\rho_{x}\left(x_{d}\right) \rho_{u}\left(x_{d}\right)+\varepsilon^{\prime}$ exists, i.e., condition (16) is satisfied. Thus, $\dot{V}_{1}\left(x_{d}\right)<0$ is achieved and the approximation error converges to zero. The closed-loop dynamics are of the form (15) and are globally asymptotically stable. Integrating (24) leads to the adaptation law in (15) for the weights of the RBF network, which ends the proof.

Remark 1. The obtained closed-loop sliding mode dynamics do not contain any matched uncertainty and reduce the influence of unmatched uncertainty. Thus, the proposed integral sliding surface with RBF networks improves the control performance of the ISMC against system uncertainty especially unmatched uncertainty. In (Cao and Xu 2004), the following closed-loop dynamics were obtained by using a basic integral sliding surface,

$$
\dot{x}_{d}=f\left(x_{d}\right)+B\left(x_{d}\right) u_{n}\left(x_{d}\right)+\left\{I-B\left(x_{d}\right)\left[D B\left(x_{d}\right)\right]^{-1} D\right\} \Delta f_{u}\left(x_{d}\right)
$$

The Euclidean norm of the uncertainty term is

$$
\begin{aligned}
& \left\|\left\{I-B\left(x_{d}\right)\left[D B\left(x_{d}\right)\right]^{-1} D\right\} \Delta f_{u}\left(x_{d}\right)\right\|=\left\|Q\left(x_{d}\right) \phi_{u}\left(x_{d}\right)\right\| \\
& =\left\|Q_{1}\left(x_{d}\right) \phi_{u 1}\left(x_{d}\right)+Q_{2}\left(x_{d}\right) \phi_{u 2}\left(x_{d}\right)\right\| \geq\left\|Q_{2}\left(x_{d}\right) \phi_{u 2}\left(x_{d}\right)\right\|
\end{aligned}
$$

Note that the last term in (28) is the norm of the uncertainty term in (16) of the developed method. Therefore, the developed method has a more robust dynamics to the unmatched uncertainty than the method in (Cao and Xu 2004).

Remark 2. Additionally, Castanos and Fridman (2006) pointed out that $\left\|I-B[D B]^{-1} D\right\|=1$ when the input matrix $B$ is constant and the design matrix is selected as $D=B^{+}$( $B^{+}$is the left inverse of $B$, i.e. $B^{+}=\left[B^{T} B\right]^{-1} B^{T}$ ). Then the closed-loop dynamics of the sliding surface in Castanos and Fridman (2006) are

$$
\dot{x}_{d}=f\left(x_{d}\right)+B u_{n}\left(x_{d}\right)+\Delta f_{u}\left(x_{d}\right)
$$

For the closed-loop dynamics obtained using the proposed integral sliding surface in this paper, with the same $B$ and $D$, the norm of the unmatched uncertainty is

$$
\left\|\Delta f_{u}\left(x_{d}\right)\right\|=\left\|Q\left(x_{d}\right) \phi_{u}\left(x_{d}\right)\right\|=\left\|Q_{1}\left(x_{d}\right) \phi_{u 1}\left(x_{d}\right)+Q_{2}\left(x_{d}\right) \phi_{u 2}\left(x_{d}\right)\right\| \geq\left\|Q_{2}\left(x_{d}\right) \phi_{u 2}\left(x_{d}\right)\right\|
$$

Therefore, the norm of the unmatched uncertainty in the closed-loop dynamics is further reduced by the developed method compared with the method in Castanos and Fridman (2006).

\section{Sliding Mode Control Law}

In the integral-type sliding mode control, a control law is usually designed in the following form

$$
u(t)=\left\{\begin{array}{lll}
u_{n}(x)-\rho(x) \frac{[D B(x)]^{T} S(x)}{\left\|[D B(x)]^{T} S(x)\right\|} & \text { if } \quad S(x) \neq 0 \\
u_{n}(x) & \text { if } \quad S(x)=0
\end{array}\right.
$$

Here, $u_{n}(x)$ is the nominal control of the nominal nonlinear plant (2). When $S(x)$ does not equal to zero, the nominal control will be modified by the second term in the above equation, which is a normalized product of sliding vector with DB and D can be designed as $D=B^{+}$in Remark 2. 
Theorem 2. The closed-loop state is guaranteed to be maintained on the proposed sliding mode (5) by the control law in (31) for the switching gain satisfies the following condition

$$
\rho(x)>\frac{1}{\varepsilon_{b}}\left\{\left(1-\varepsilon_{b}\right)\left\|u_{n}(x)\right\|+\rho_{m}(x)+\left\|[D B(x)]^{-1} D\right\| \rho_{u}(x)+\left\|[D B(x)]^{-1}\right\| \cdot\|g(x)\|\right\}
$$

with the vector $g(x)$ described in (7).

Proof. Define a Lyapunov function as below,

$$
V_{2}(x)=\frac{1}{2} S^{T}(x) S(x)
$$

Substituting the control law in (31) under the condition $S(x) \neq 0$ and considering the uncertainty bounds, its first derivative with respect to time $t$ is expressed as

$$
\begin{aligned}
& \dot{V}_{2}(x)=S^{T}(x) \dot{S}(x) \\
& =-\rho(x)\left\|[D B(x)]^{T} S(x)\right\|-\Delta B_{m}(x) \rho(x)\left\|[D B(x)]^{T} S(x)\right\|+S^{T}(x) D B(x) \Delta B_{m}(x) u_{n}(x) \\
& +S^{T}(x) D B(x) \Delta f_{m}(x)+S^{T}(x)[D B(x)] \cdot[D B(x)]^{-1} D \Delta f_{u}(x)-S^{T}(x)[D B(x)] \cdot[D B(x)]^{-1} g(x) \\
& \leq\left\{\begin{array}{l}
-\rho(x)-\Delta B_{m}(x) \rho(x)+\left\|\Delta B_{m}(x) u_{n}(x)\right\|+\left\|\Delta f_{m}(x)\right\| \\
+\left\|[D B(x)]^{-1} D\right\| \cdot\left\|\Delta f_{u}(x)\right\|+\left\|[D B(x)]^{-1}\right\| \cdot\|g(x)\|
\end{array}\right\}\left\|[D B(x)]^{T} S(x)\right\| \\
& \leq-\left\{\begin{array}{l}
\varepsilon_{b} \rho(x)-\left(1-\varepsilon_{b}\right)\left\|u_{n}(x)\right\|-\rho_{m}(x) \\
-\left\|[D B(x)]^{-1} D\right\| \rho_{u}(x)-\left\|[D B(x)]^{-1}\right\| \cdot\|g(x)\| \|^{2}
\end{array}\right\}\left\|[D B(x)]^{T} S(x)\right\|<0
\end{aligned}
$$

Since $D B(x)$ is full column rank and $S(x) \neq 0$, the switching gain $\rho(x)$ satisfying (32) ensures the above inequality in (34), Thus, condition in (32) guarantees that the closed-loop state is maintained on the proposed sliding mode by the control law in (31).

Remark 3: The control law (31) ensures the system state to be maintained on the sliding surface even the unmatched uncertainty is not completely compensated for by the adaptive network. As long as the switching gain is high enough to satisfy the condition (32), the system stability is guaranteed.

\section{Numerical Example}

Consider the parameters of a nonlinear uncertain system (1) as follows.

$$
f(x)=\left[\begin{array}{ccc}
0 & 0 & 1 \\
0 & -2 & 0 \\
-1 & 0 & 2
\end{array}\right]\left[\begin{array}{l}
x_{1} \\
x_{2} \\
x_{3}
\end{array}\right], \quad B(x)=\left[\begin{array}{cc}
1 & 0.1 \sin ^{2}\left(x_{1}\right)-2 \\
0.1 \cos ^{3}\left(x_{2}\right)-3 & 4 \\
5 & 6+0.1 \cos \left(x_{3}^{4}\right)
\end{array}\right]
$$

The matched and unmatched uncertainties are of the following form.

$$
\begin{aligned}
& \Delta f_{m}(x)=\left[\begin{array}{c}
0.1 x_{1}^{2}+0.1 \\
0.1 x_{1}^{2}+0.2 x_{2}^{3}+0.1 x_{3}^{4}+0.1
\end{array}\right], \quad \Delta B_{m}(x)=\left[\begin{array}{cc}
0.1 \sin \left(x_{2}\right) & 0 \\
0 & 0.1 \cos ^{2}\left(x_{1}\right)
\end{array}\right] \\
& \Delta f_{u}(x)=\left[\begin{array}{lll}
0.5 x_{1} \cos \left(x_{2}\right) & 0.2 x_{2} \sin \left(x_{1}^{2}\right) & 0.4 x_{3} \sin ^{2}\left(x_{2}\right)
\end{array}\right]^{T} .
\end{aligned}
$$

The bounds of the matched uncertainty are $\rho_{m}(x)=0.2 x_{1}^{2}+0.2\left|x_{2}\right|^{3}+0.1 x_{3}^{4}+0.2$ and $\varepsilon_{b}=0.9$. The bound of the unmatched uncertainty is $\rho_{u}(x)=0.5\|x\|$. The eigenvalues are placed at $s=\left[\begin{array}{lll}-1 & -2 & -3\end{array}\right]^{T}$. Choose the design matrix $D=\left[\begin{array}{ccc}0 & 0.1 & 0.2 \\ 0.4 & -0.1 & 0.2\end{array}\right]$. It is then obtained that

$$
\left\|\phi_{u 2}\left(x_{d}\right)\right\| \leq\left\|\phi_{u}\left(x_{d}\right)\right\| \leq\left\|I-B\left(x_{d}\right)\left[D B\left(x_{d}\right)\right]^{-1} D\right\| \rho_{u}\left(x_{d}\right)<1.6 \times 0.5\left\|x_{d}\right\|=0.8\left\|x_{d}\right\| .
$$


Considering $\left\|B\left(x_{d}\right)\right\|$ is bounded and $\varepsilon$ is a positive constant, $\left\|B\left(x_{d}\right)\right\| \varepsilon$ is also a positive constant. Choose $\beta=\left\|x_{d}\right\|$, then $\gamma\left(\left\|x_{d}\right\|\right)=\left\|x_{d}^{T}\right\|\left\|x_{d}\right\|$. So that the condition (17) is satisfied as follows,

$$
\left\|\phi_{u 2}\left(x_{d}\right)\right\|+\left\|B\left(x_{d}\right)\right\| \varepsilon<0.8\left\|x_{d}\right\|+\left\|B\left(x_{d}\right)\right\| \varepsilon<\left\|x_{d}\right\| \text { when } x_{d} \neq 0
$$

As for the RBF structure, the network inputs are selected as $x(t)=\left[\begin{array}{lll}x_{1}(t) & x_{2}(t) & x_{3}(t)\end{array}\right]^{T}$ and are scaled to the range of $(0,1)$ before they are fed into the networks. The network centers and widths are chosen to be constant using the $K$-means clustering method and $P$-nearest center rule. Different orders and numbers of hidden nodes have been tried in the experiments and a firstorder structure with 12 hidden nodes is selected. The adaptation parameter is selected as $\eta=1$. The weights are initialized with small random values.

The simulation is run from an initial value of the state $x\left(t_{0}\right)=\left[\begin{array}{lll}0 & -2 & 1\end{array}\right]^{T}$ with a fixed step of 1 ms. Figure.1 shows three system responses: the dotted line denotes the idea system without any uncertainties; the solid line denotes the system under the proposed control; dash-dotted line is by the system under conventional ISMC. It is evident that the proposed method has a response much closer to the idea response than the ISMC in (Cao and Xu 2004). To show the network convergence, the three estimation errors $\left(e_{1}, e_{2}, e_{3}\right)$ are displayed in Figure.2. It is seen in Figure.2 that all the three errors converge to zero, which implies that the networks are adapted to represent the transforms of partial unmatched uncertainties.
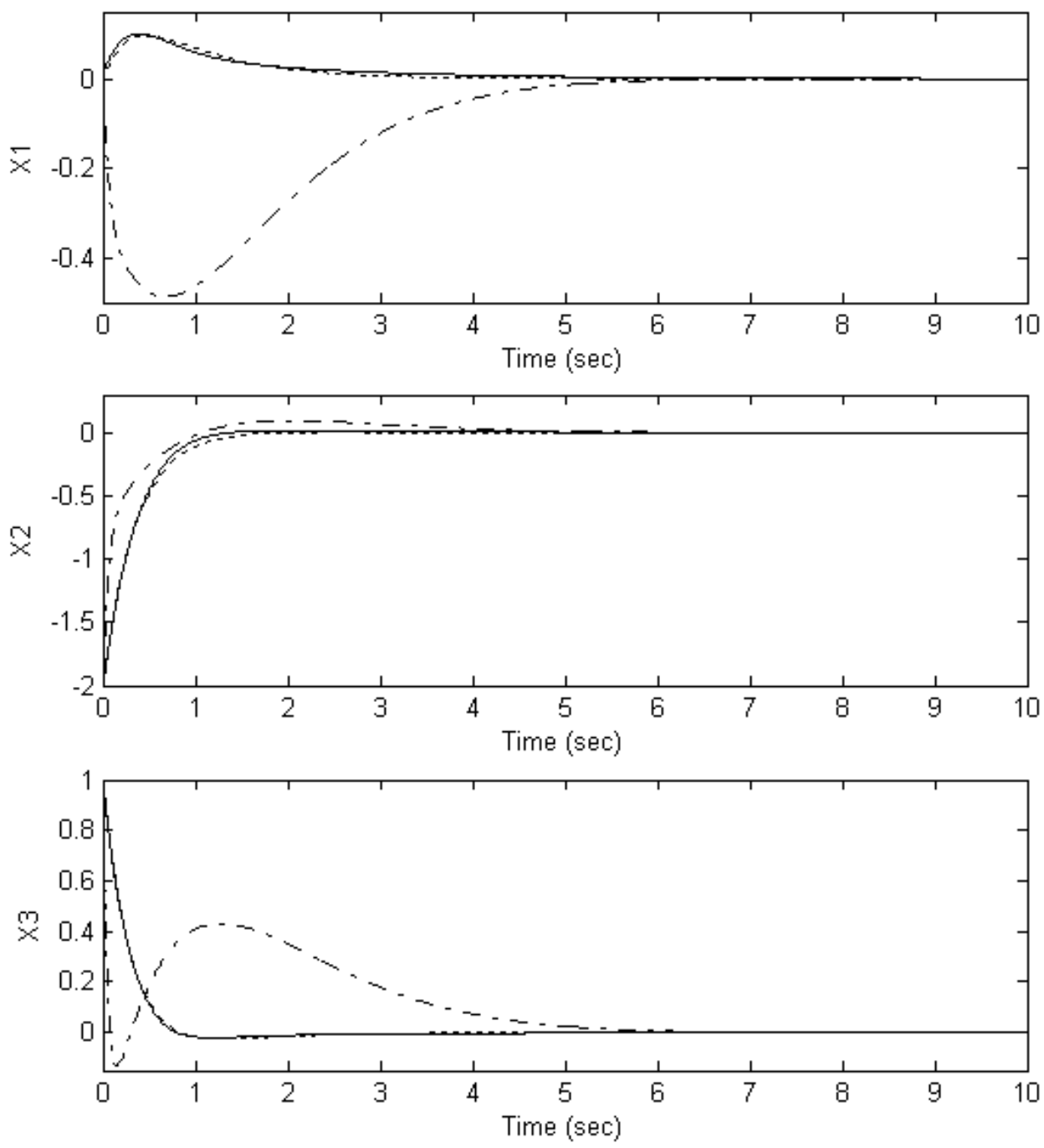

Figure1. The response of the state $x(t)$ : dotted line --- ideal system (without any uncertainty) response, solid line --- response under the proposed control, dash-dotted line --- response using the existing ISMC in (Cao and Xu 2004) 


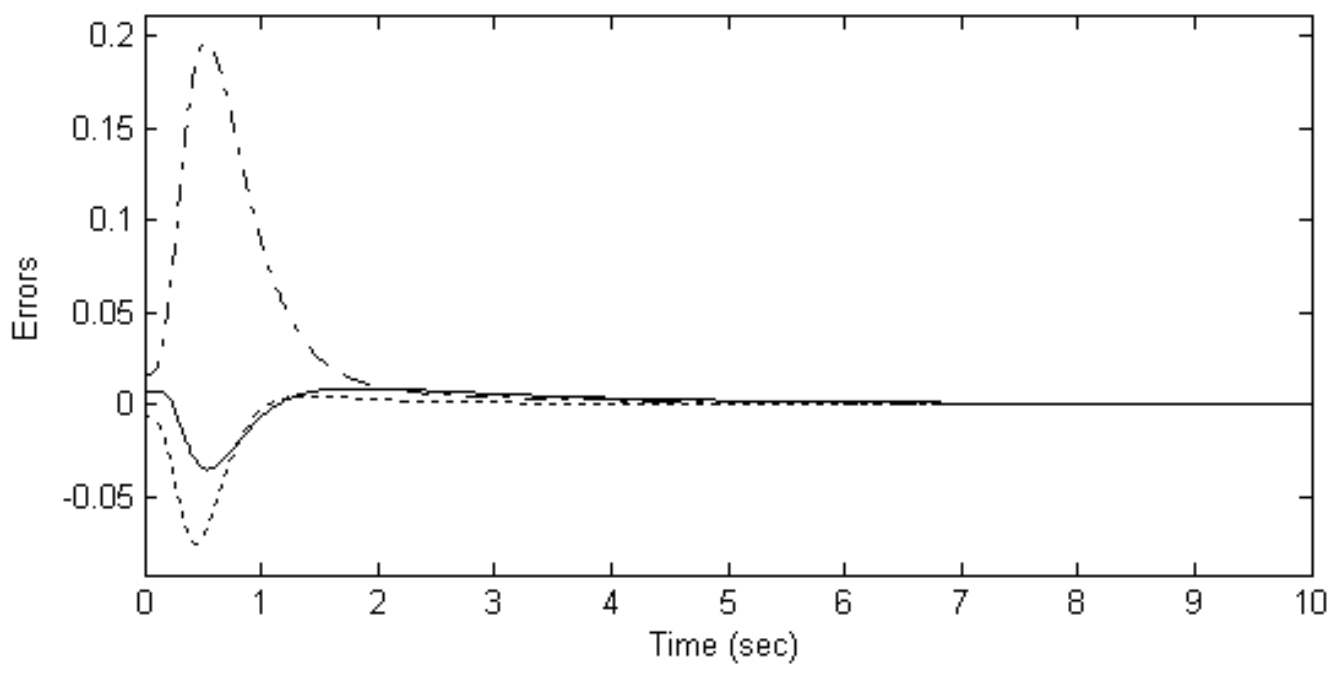

Figure 2. The approximation errors: solid line--- $e_{1}$, dash-dotted line--- $e_{2}$, dotted line--- $e_{3}$

\section{Conclusions}

A new integral sliding mode control scheme with an adaptive RBF network is proposed, which eliminates completely the matched uncertainties and partially the unmatched uncertainty in the resultant system closed-loop dynamics. Enhanced robustness to the unmatched uncertainties is proved by reduced norm of these uncertainties appeared in the closed-loop dynamics compared with the existing methods. The method is realized using the approximation feature of RBF neural networks and Lyapunov theory. The new selection condition for the switching gain is derived to ensure the system states being maintained on the proposed sliding surface. Numerical simulations showed the network approximation and superior the proposed method to the existing methods.

\section{References}

Cao, W.-J. and Xu, J.-X. 2004. Nonlinear integral-type sliding surface for both matched and unmatched uncertain systems. IEEE Trans. Automatic Control, Vol. 49, pp. 1335-1360.

Castanos, F. and Fridman, L. 2006. Analysis and design of integral sliding manifolds for systems with unmatched perturbations. IEEE Trans. Automatic Control, Vol. 51, pp. 853-858.

Fridman, L., Poznyak, A. and Bejarano, F. 2005. Decomposition of the min-max multi-model problem via integral sliding mode. Int. J. Robust Nonlinear Control, Vol. 15, pp. 559-574.

Funahashi, K. 1989. On the approximate realization of continuous mappings by neural networks. Neural Networks, Vol. 2, pp. 183-192.

Matthews, G. P. and DeCarlo, R. A. 1988. Decentralized tracking for a class of interconnected nonlinear systems using variable structure control. Automatica, Vol. 24, pp. 187-193.

Niu, Y., Ho, D. W. C. and Lam J. 2005. Robust integral sliding mode control for uncertain stochastic systems with time-varying delay. Automatica, Vol. 41, pp. 873-880.

Poznyak, A., Fridman, L. and Bejarano, F. J. 2004. Mini-max integral sliding-mode control for multimodel linear uncertain systems. IEEE Trans. Automatic Control, Vol. 49, pp. 97-102.

Sanner, R. M. and Slotine, J. -J. E. 1992. Gaussian networks for direct adaptive control. IEEE Trans. Neural Networks, Vol. 3, pp. 837-863.

Seok P.B.; Yoo S.J.; Park J.B.; Choi Y.H. 2009. Adaptive Neural Sliding Mode Control of Nonholonomic Wheeled Mobile Robots With Model Uncertainty, IEEE Trans. Control Systems Technology, Vol.17, No.1, pp. 135-146.

Utkin, V. I. 1977. Variable structure systems with sliding modes. IEEE Trans. Automatic Control, Vol. 22, pp. 212-222.

Utkin, V. and Shi, J. 1996. Integral sliding mode in systems operating under uncertainty conditions. Proc. Conf. Decision and Control, Kobe, Japan, pp. 4591-4596.

Wang, C. and Hill, D. J. 2006. Learning from neural control. IEEE Trans. Neural Networks, Vol. 17, pp. 130-146.

Wang, S.W. and Yu, D.L. 2008, Neural Network-Based Integral Sliding Mode Control for Nonlinear Uncertain Systems (Lecture Notes in Electrical Engineering, Advances in Industrial Engineering and Operations Research, Springer Verlag. 
Yinxing H.; Yi J.; Zhao D.; Qian D. 2008. Robust control using incremental sliding mode for underactuated systems with mismatched uncertainties, Proc. of American Control Conference, Vol.3, pp. 2364-2372.

Young, K. D., Utkin, V. I. and Ozguner, U. 1999. A control engineer's guide to sliding mode control. IEEE Trans. Control Systems Technology, Vol. 7, pp. 328-342.

\section{Biographical notes}

Dr. S. W. Wang is with the Yuan Hang Scientific Corp. Weihai City, CHINA

Dr. D.W. Yu is with the Department of Engineering, Northeast University at Qinhuangdao, CHINA

Professor Dingli Yu received B.Eng from Harbin Civil Engineering College, China in 1982, M.Sc from Jilin University of Technology (JUT), China in 1986, and the PhD from Coventry University, U.K. in 1995, all in Control Engineering. Dr. Yu was a lecturer at JUT from 1986 to 1990, a visiting researcher at University of Salford, U.K. in 1991, a post-doctoral research fellow at Liverpool John Moores University (LJMU) from 1995 to 1998. He joined LJMU Engineering School in 1998 as a Senior Lecturer and was promoted to a Reader in 2003, then to Professor of Control Systems in 2006. He is the associate editor of two journals, International Journal of Modelling Identification and Control and International Journal of Information \& Systems Sciences. He organized two special issues in 2006, "Fault Detection, Diagnosis and Fault Tolerant Control for Dynamic Systems" and "Intelligent Monitoring and Control for Industrial systems". He serves as a member of the IFAC SAVEPROCESS Committee, and has been IPC member for many international conferences. He is a fellow of IET and Senior Member of IEEE. He leads the Control Systems Research group at LJMU. His current research interests include fault detection and fault tolerant control of bilinear and nonlinear systems, adaptive neural networks and their control applications, model predictive control for chemical processes and automotive engines and real-time evaluations, in these areas he has published more than 160 journal and conference papers.

Received December 2010

Accepted April 2011

Final acceptance in revised form July 2011 